\title{
Optimisation Approach for Parameter Estimation of the Generalised PTT Viscoelastic Model *
}

\author{
M. Fernanda P. Costa $(\bowtie)^{1[0000-0001-6235-286 X]}$, C. \\ Coelho ${ }^{1[0000-1111-2222-3333]}$, and L.L. Ferrás ${ }^{1[0000-0001-5477-3226]}$ \\ Centre of Mathematics \\ University of Minho, Campus de Gualtar 4710 - 057 Braga, Portugal \\ mfc@math.uminho.pt, ceciliaeduarda58@gmail.com, luislimafr@gmail.com \\ https://www.cmat.uminho.pt/
}

\begin{abstract}
The exponential form of the original Phan-Thien and Tanner (PTT) model is often used to study complex viscoelastic fluids. Recently, a generalised version of the PTT model, that uses the Mittag-Leffler function to compute a new function of the trace of the stress tensor, was proposed. This new model adds one or two additional fitting parameters that allow for greater fitting capability. In this paper, we propose two optimisation problems for estimating the model parameters when fitting experimental data in shear (storage modulus, loss modulus, shear viscosity). We also propose a numerical sequential approach for solving one of these problems. The optimal values for the parameters produced by the optimisation approach allow the model to reproduce almost exactly the experimental data.
\end{abstract}

Keywords: Optimisation - Sequential Approach · Viscoelastic Models · Phan-Thien and Tanner $\cdot$ gPTT $\cdot$ Rheology

\section{Introduction}

The stress felt in our hands $(\sigma)$, induced by stretching a spring is proportional to the deformation $\left(\gamma_{e}\right)$ with $G_{0}$ the constant of proportionality. This is known as Hook's law (see the illustration in Fig. 1 - left). The stress felt by stretching a dashpot is proportional to the velocity of that deformation $\left(\frac{d \gamma_{f}}{d t}\right)$, with $\eta$ the constant of proportionality. This is known as Newton's law for fluids. In the Newton's law, a smooth stretch leads to a low stress and a rapid stretch leads to a high stress (see the illustration in Fig. 1 - centre). The Newton's law for fluids states that the faster we move our hands, the higher the resistance.

These are two laws universally accepted and observed experimentally (under the limitations of the experiment and the material used).

\footnotetext{
* Supported by FCT - Fundação para a Ciência e a Tecnologia, through projects UIDB/00013/2020 and UIDP/00013/2020, and CMAT - Centre of Mathematics, University of Minho.
} 
The Maxwell model of viscoelasticity can be easily obtained by combining a spring and a dashpot in series, as shown in Fig. 1.

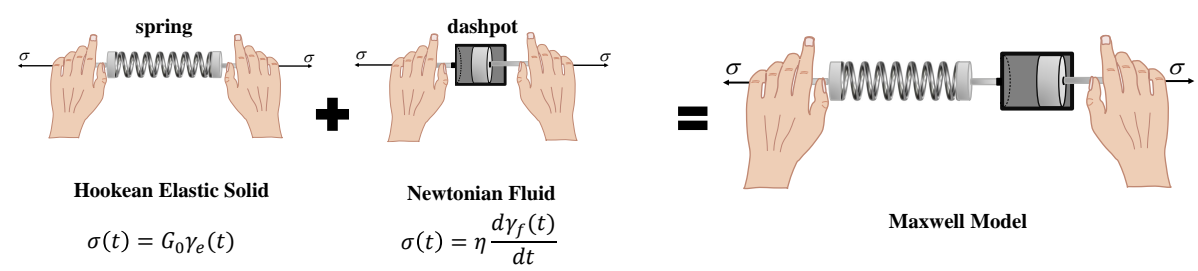

Fig. 1. The Maxwell model represented by a combination of a spring and a dashpot, in series. The subscripts $e$ and $f$ stand for elastic solid and fluid, respectively.

To derive the Maxwell model one assume that the total deformation, $\gamma$, is the sum of the deformation applied to the spring, $\gamma_{e}$, and the deformation applied to the dashpot, $\gamma_{f}$. That is, $\gamma(t)=\gamma_{e}(t)+\gamma_{f}(t)$. Applying the time derivative to both sides of this equation, we obtain the total rate of deformation $\frac{d \gamma(t)}{d t}=$ $\frac{d \gamma_{e}(t)}{d t}+\frac{d \gamma_{f}(t)}{d t}$. Applying again the time derivative to both sides of Hook's law and substituting in the equation for the total rate of deformation, we arrive at the Maxwell model:

$$
\sigma(t)+\lambda \frac{d \sigma(t)}{d t}=\eta \frac{d \gamma(t)}{d t},
$$

with $\lambda=\frac{\eta}{G_{0}}$ the relaxation time of the fluid (in seconds), $\eta$ the zero shear-rate viscosity (the viscosity obtained when $\frac{d \gamma(t)}{d t} \rightarrow 0$, in Pascal $\times$ seconds), and $\lambda, \eta \geq 0$. The model can also be written in integral form as:

$$
\sigma(t)=\int_{0}^{t} G_{0} e^{-\frac{t-t^{\prime}}{\lambda}} \frac{d \gamma}{d t^{\prime}} d t^{\prime} .
$$

This model is really simple and lacks a generalisation to every point in the three-dimensional (3D) space $\boldsymbol{x}=(x, y, z)$. The state of stress of a point in the $3 \mathrm{D}$ space can be represented by a $3 \times 3$ tensor (with 9 stress components),

$$
\boldsymbol{\sigma}(t, \boldsymbol{x}) \equiv \boldsymbol{\sigma}=\left[\begin{array}{lll}
\sigma_{x x} & \sigma_{x y} & \sigma_{x z} \\
\sigma_{y x} & \sigma_{y y} & \sigma_{y z} \\
\sigma_{z x} & \sigma_{z y} & \sigma_{z z}
\end{array}\right],
$$

and the rate of deformation of a body of viscoelastic material in the 3D space, $\frac{d \gamma(t)}{d t}$, is related to the way the velocity of a material point varies in that space (velocity gradient $\nabla \boldsymbol{u}$ ) [1] by, 


$$
\frac{d \gamma(t)}{d t}=2 \boldsymbol{D}=\left(\nabla \boldsymbol{u}+(\nabla \boldsymbol{u})^{T}\right)=\left[\begin{array}{lll}
\frac{\partial u}{\partial x}+\frac{\partial u}{\partial x} & \frac{\partial u}{\partial y}+\frac{\partial v}{\partial x} & \frac{\partial u}{\partial z}+\frac{\partial w}{\partial x} \\
\frac{\partial u}{\partial y}+\frac{\partial v}{\partial x} & \frac{\partial v}{\partial y}+\frac{\partial v}{\partial y} & \frac{\partial v}{\partial z}+\frac{\partial w}{\partial y} \\
\frac{\partial u}{\partial z}+\frac{\partial w}{\partial x} & \frac{\partial v}{\partial z}+\frac{\partial w}{\partial y} & \frac{\partial w}{\partial z}+\frac{\partial w}{\partial z}
\end{array}\right]
$$

where $\boldsymbol{u}=(u, v, w)$ is the velocity vector, $\nabla$ is the gradient operator and $\boldsymbol{D}(t, \boldsymbol{x}) \equiv \boldsymbol{D}$ is the rate of deformation tensor. Note that this derivation is not trivial. More details can be found in any classical book of fluid or solid mechanics [1].

This generalisation to the 3D space results in the Maxwell model,

$$
\boldsymbol{\sigma}+\lambda \frac{d \boldsymbol{\sigma}}{d t}=2 \eta \boldsymbol{D}
$$

that is only valid for really small deformations.

Qualitative and quantitative descriptions of physical phenomena must remain unchanged if we make any change in the point of view from which we observe them. This is called objectivity or frame-invariance. The Maxwell model is not invariant and therefore cannot be used in large deformations. This is due to the operator $\frac{d \boldsymbol{\sigma}}{d t}$. This time derivative is not invariant, and therefore the term $\frac{d \boldsymbol{\sigma}}{d t}$ should be replaced by some physically equivalent term. The new term should provide the time variation of the stress tensor, and also guarantee that this variation is always the same for all observers. A possible solution is to use:

1. Upper-Convected time derivative:

$$
\stackrel{\nabla}{\boldsymbol{\sigma}}=\frac{\partial}{\partial t} \boldsymbol{\sigma}+(\mathbf{v} \cdot \nabla) \boldsymbol{\sigma}-(\nabla \mathbf{v})^{T} \cdot \boldsymbol{\sigma}-\boldsymbol{\sigma} \cdot(\nabla \mathbf{v})
$$

with

$$
\frac{D}{D t} \boldsymbol{\sigma}=\frac{\partial \boldsymbol{\sigma}}{\partial t}+\boldsymbol{u} \cdot \nabla \boldsymbol{\sigma}
$$

known as the material derivative. This derivative describes the time rate of change of some physical quantity of a material element that is subjected to a space-and-time-dependent macroscopic velocity field. In Eq. (2), the "." represents the usual dot product and the term $(\mathbf{v} \cdot \nabla) \boldsymbol{\sigma}$ results in a tensor with entry $i, j$ given by,

$$
u \frac{\partial \sigma_{i j}}{\partial x}+v \frac{\partial \sigma_{i j}}{\partial y}+w \frac{\partial \sigma_{i j}}{\partial z}
$$

where $i=x, y, z$ and $j=x, y, z$.

2. Lower-Convected time derivative:

$$
\stackrel{\Delta}{\boldsymbol{\sigma}}=\frac{\partial}{\partial t} \boldsymbol{\sigma}+(\mathbf{v} \cdot \nabla) \boldsymbol{\sigma}+\boldsymbol{\sigma} \cdot(\nabla \mathbf{v})^{T}+(\nabla \mathbf{v}) \cdot \boldsymbol{\sigma}
$$

3. Gordon-Schowalter time derivative: 


$$
\stackrel{\circ}{\boldsymbol{\sigma}}=\frac{\partial}{\partial t} \boldsymbol{\sigma}+(\mathbf{u} \cdot \nabla) \boldsymbol{\sigma}-(\nabla \mathbf{u})^{T} \cdot \boldsymbol{\sigma}-\boldsymbol{\sigma} \cdot(\nabla \mathbf{u})+\xi(\boldsymbol{\sigma} \cdot \mathbf{D}+\mathbf{D} \cdot \boldsymbol{\sigma}),
$$

where $\xi$ is a real parameter typically set in $[0,1]$. The $\xi$ parameter accounts for the slip between the molecular network and the continuous medium.

Note that a combination of Upper-Convected, Lower-Convected and Gordon-Schowalter derivatives, can also be used.

The Upper-Convected Maxwell (UCM) model is therefore a generalisation of the Maxwell material for the case of large deformations using the upperconvected time derivative. Note that the Lower-Convected Maxwell model can be obtained in a similar way.

The UCM model was proposed by James G. Oldroyd, and can be written as:

$$
\boldsymbol{\sigma}+\lambda \stackrel{\nabla}{\sigma}=2 \eta \mathbf{D}
$$

This model is frame-invariant and can be used to model large deformations. The choice of derivative will influence the characteristics of the model, and its ability to model real viscoelastic materials.

The Maxwell and the UCM models were obtained mechanically from springs and dashpots but can also be derived from molecular theory.

In the recent decades, several models were developed, either based on mechanical analogues of springs and dashpots, molecular theory, or, network theories. Network theories characterise the materials as networks of entangled molecules (see Fig. 2).

In the 1970's a model was proposed in the literature that is apparently quite similar to the UCM model but that can better model real fluids.

It was derived from a Lodge-Yamamoto type of network theory (see Fig. 2) for polymeric fluids [2-5]. For the isothermal case, the model can be expressed by the constitutive equation

$$
K\left(\boldsymbol{\sigma}_{k \boldsymbol{k}}\right) \boldsymbol{\sigma}+\lambda \stackrel{\circ}{\boldsymbol{\sigma}}=2 \eta \mathbf{D}
$$

where $\boldsymbol{\sigma}_{\boldsymbol{k} \boldsymbol{k}}$ is the trace of the stress tensor and $K\left(\boldsymbol{\sigma}_{\boldsymbol{k} \boldsymbol{k}}\right)$ is the network destruction function given by either a linear or an exponential function of $\sigma_{k \boldsymbol{k}}$. The only difference to the UCM model (Eq. (4)) is the presence of $K\left(\boldsymbol{\sigma}_{\boldsymbol{k} \boldsymbol{k}}\right)$ and the use of the Gordon-Schowalter time derivative. 


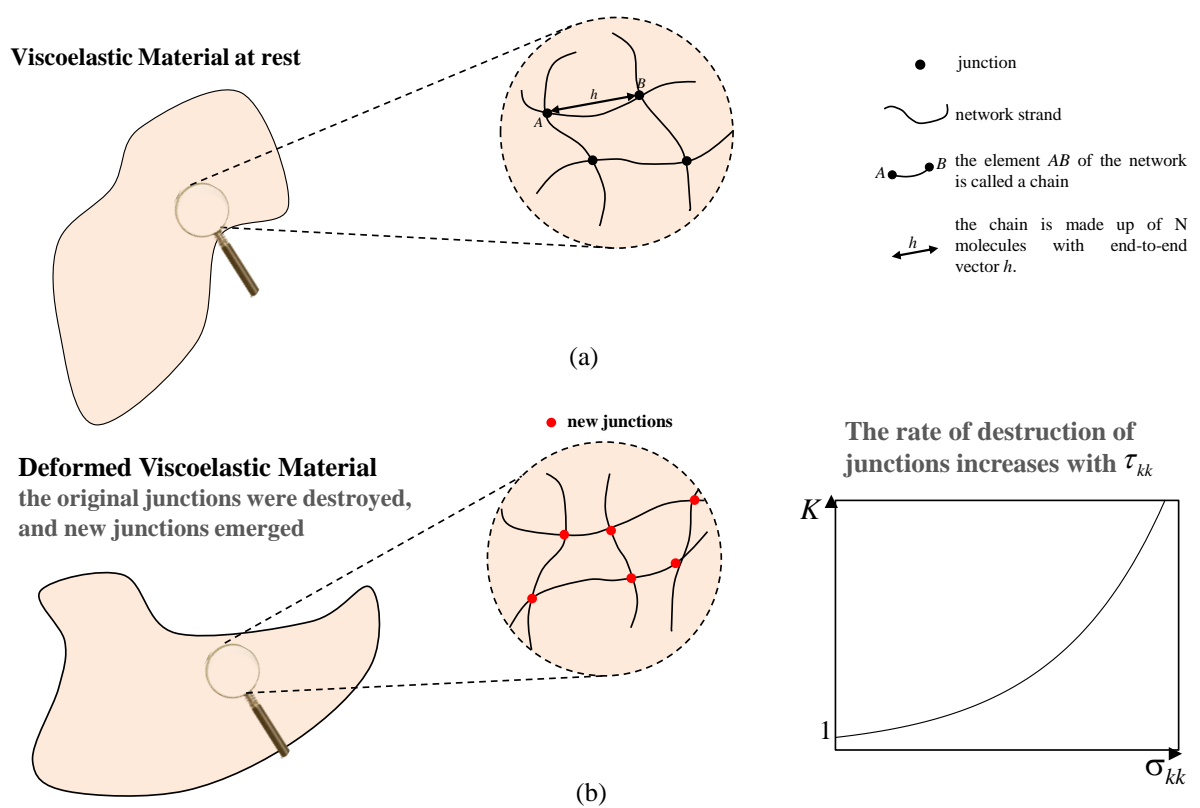

Fig. 2. The lodge-Yamamoto theory. (a) The molecules entanglements are represented by a network strand connected by junctions. (b) These junctions are destroyed with deformation of the material and new junctions are created. Note that the rate of destruction of junctions $\left(K\left(\sigma_{k k}\right)\right)$ is represented schematically in the graph shown in the left bottom corner. $K$ increases with the stress.

More recently (2019), a generalised Phan-Thien - Tanner constitutive equation (gPTT) was proposed [5], where $K\left(\sigma_{k k}\right)$, the network destruction function is defined by,

$$
K\left(\sigma_{k k}\right)=\Gamma(\beta) E_{\alpha, \beta}\left(\frac{\varepsilon \lambda}{\eta} \sigma_{k k}\right)
$$

where $\alpha, \beta$ and $\varepsilon$ are real positive parameters, being $\varepsilon$ known by the extensibility parameter (in the classical model). Here the normalisation $\Gamma(\beta)$ is used to ensure that $K(0)=1$ for all choices of the parameter $\beta . E_{\alpha, \beta}($.$) is the generalised$ Mittag-Leffler function [6]

$$
E_{\alpha, \beta}(z)=\sum_{k=0}^{\infty} \frac{z^{k}}{\Gamma(\alpha k+\beta)}
$$

and $\Gamma$ is the Gamma function given by

$$
\Gamma(t)=\int_{0}^{\infty} x^{t-1} e^{-x} d x .
$$

We note that, for $\alpha=\beta=1$ the Mittag-Leffler function $E_{\alpha, \beta}($.$) reduces to the$ exponential function. 
The parameters $\alpha, \beta, \varepsilon, \lambda, G_{0}$ and $\xi$ will affect the behaviour of the constitutive model (recall that $G_{0}=\eta / \lambda$ ). The proposed mathematical model depends on six unknown parameters that require investigation. Since the mathematical model simulates real fluids, the parameter values can be estimated based on experimental data. The aim of a parameter estimation optimisation problem is to calibrate the model so that it can reproduce the experimental data. This is performed by minimising an objective function that measures the goodness of the fit. Therefore, it is important to develop an optimisation methodology to obtain the fitting parameters correctly, considering different flows.

Since this model has only recently been proposed in the literature, there is no specific optimisation approach for this type of model. Therefore, the aim of this work is to develop a new optimisation approach that consider weak flows.

This work is organised as follows. In Section 2, we introduce the governing equations for an isothermal incompressible fluid described by the generalised PTT model (gPTT), and derive the equations for the storage and loss moduli and the shear viscosity. In Section 3, we present the optimisation problems for estimating the parameters of the model. In Section 4, we describe the optimisation approach for solving the optimisation problems. We also show that the optimal parameter values allowed the model to reproduce almost exactly the experimental data. The paper ends with the conclusions in Section 5.

\section{Equations}

In this section we describe the governing equations for an isothermal incompressible fluid described by the gPTT model, and the equations for the storage and loss moduli and the shear viscosity.

\subsection{Governing Equations}

The equations governing the flow of an isothermal incompressible fluid are the continuity equation,

$$
\nabla \cdot \mathbf{u}=0
$$

and the momentum equation,

$$
\rho \frac{D \mathbf{u}}{D t}=-\nabla p+\nabla \cdot \boldsymbol{\sigma}
$$

together with the constitutive equation,

$$
\Gamma(\beta) E_{\alpha, \beta}\left(\frac{\varepsilon \lambda}{\eta} \sigma_{k k}\right) \boldsymbol{\sigma}+\lambda \stackrel{\circ}{\sigma}=2 \eta \mathbf{D}
$$

where $p$ is the pressure, $t$ the time and $\rho$ the mass density. The Gordon-Schowalter derivative $\stackrel{\boldsymbol{\sigma}}{ }$ is given by Eq. (3). 


\subsection{The Storage and Loss Moduli}

The storage and loss moduli [7] allow one to study how our model behaves when an oscillatory deformation is imposed. We can see how much is recovered (Hook's law) and how much is lost (Newton's law) when the deformation is applied. The storage and loss are represented by $G^{\prime}$ and $G^{\prime \prime}$ respectively.

The $G^{\prime}$ and $G^{\prime \prime}$ can be determined using Laplace Transform, $\mathcal{L}$, and the convolution theorem. Let $\bar{\sigma}(s)$ and $\bar{\gamma}(s)$ denote the Laplace transform of $\sigma(t)$ and $\gamma(t)$, respectively. For small deformations, the gPTT model reduces to the integral Maxwell model (Eq. (1)) and we have that (assuming $\sigma(t)=0$ for $t=0$ ):

$$
\begin{aligned}
\bar{\sigma}(s) & =\mathcal{L}\{\sigma(t)\} \\
& =\mathcal{L}\left\{\int_{0}^{t} G_{0} e^{-\frac{t-t^{\prime}}{\lambda}} \frac{d \gamma}{d t^{\prime}} d t^{\prime}\right\} \\
& =\int_{0}^{\infty} G_{0} e^{-\frac{t}{\lambda}} e^{-s t} d t \int_{0}^{\infty} \frac{d \gamma(t)}{d t} e^{-s t} d t \\
& =\frac{s G_{0} \lambda \bar{\gamma}(s)}{1+s \lambda} .
\end{aligned}
$$

The ratio of $\bar{\sigma}(s)$ and $\bar{\gamma}(s)$ is given by:

$$
\frac{\bar{\sigma}(s)}{\bar{\gamma}(s)}=\frac{s G_{0} \lambda}{1+s \lambda}=G^{*}(s)
$$

where $\bar{\sigma}(s)$ and $\bar{\gamma}(s)$ are proportional, as in Hook's law, and therefore the symbol $G^{*}$ is used to represent this modulus.

Assuming that $s=i \omega(i=\sqrt{-1})$, we have that,

$$
G^{*}(i \omega)=\frac{G_{0}(\lambda \omega)^{2}}{1+(\lambda \omega)^{2}}+i \frac{G_{0} \lambda \omega}{1+(\lambda \omega)^{2}}=G^{\prime}+i G^{\prime \prime}
$$

\subsection{Weak Steady Flows}

For steady simple shear flows, the explicit expressions for viscosity are easily

found. Following the work of Alves et al. [8] and considering a simple plane shear flow aligned with the $x$-axis, the constitutive equation (5) reduces to:

$$
\begin{gathered}
K\left(\sigma_{k k}\right) \sigma_{x x}=(2-\xi)(\lambda \dot{\gamma}) \sigma_{x y}, \\
K\left(\sigma_{k k}\right) \sigma_{y y}=-\xi(\lambda \dot{\gamma}) \sigma_{x y}, \\
K\left(\sigma_{k k}\right) \sigma_{x y}=G_{0} \lambda \dot{\gamma}+(1-\xi / 2)(\lambda \dot{\gamma}) \sigma_{y y}-\frac{\xi}{2}(\lambda \dot{\gamma}) \sigma_{x x},
\end{gathered}
$$


where $\dot{\gamma}$ is the constant shear rate $\dot{\gamma}=|d u / d y|$. The division of each member of Eq. (7) by the respective member of Eq. (8) leads to the relationship $\sigma_{y y}=-\sigma_{x x} \xi /(2-\xi)$, and, its substitution in Eq. (9) together with $\sigma_{x y}$ obtained from Eq. (7) and $K\left(\sigma_{k k}\right)=\Gamma(\beta) E_{\alpha, \beta}\left(\frac{\varepsilon}{G_{0}}\left(\sigma_{x x}+\sigma_{y y}\right)\right)$ leads to the following nonlinear equation for $\sigma_{x x}$ :

$$
\Gamma^{2}(\beta) E_{\alpha, \beta}\left(\frac{\varepsilon}{G_{0}}\left(\frac{2-2 \xi}{2-\xi}\right) \sigma_{x x}\right)^{2} \sigma_{x x}-(2-\xi)(\lambda \dot{\gamma})^{2}\left[G_{0}-\sigma_{x x} \xi\right]=0
$$

that can be written in a compact form as

$$
h\left(\dot{\gamma} ; \sigma_{x x}, \lambda, G_{0}, \alpha, \beta, \varepsilon, \xi\right)=0 .
$$

The shear stress $\sigma_{x y}$ as a function of $\dot{\gamma}$ is obtained from Eq. (9), and is given by:

$$
\sigma_{x y}(\dot{\gamma})=\frac{G_{0} \lambda \dot{\gamma}-\sigma_{x x} \lambda \dot{\gamma} \xi}{\Gamma(\beta) E_{\alpha, \beta}\left(\frac{\varepsilon}{G_{0}}\left(\frac{2-2 \xi}{2-\xi}\right) \sigma_{x x}\right)} .
$$

The steady shear viscosity, $\eta(\dot{\gamma})$, is given by:

$$
\eta(\dot{\gamma})=\frac{\sigma_{x y}(\dot{\gamma})}{\dot{\gamma}}
$$

\subsection{Prony Series}

Due to the different entanglement states of the molecules in the viscoelastic material, it is common to consider more than one mode to describe its behaviour. Therefore, assuming the existence of $N$ modes, the quantities of interest presented earlier are now given by:

$$
\begin{gathered}
\hat{G}^{\prime}=\sum_{k=1}^{N} \frac{G_{0_{k}}\left(\lambda_{k} \omega\right)^{2}}{1+\left(\lambda_{k} \omega\right)^{2}} \\
\hat{G}^{\prime \prime}=\sum_{k=1}^{N} \frac{G_{0_{k}} \lambda_{k} \omega}{1+\left(\lambda_{k} \omega\right)^{2}} \\
\hat{\eta}(\dot{\gamma})=\sum_{k=1}^{N} \frac{G_{0_{k}} \lambda_{k} \dot{\gamma}-\sigma_{x x_{k}} \lambda_{k} \dot{\gamma} \xi_{k}}{\dot{\gamma} \Gamma\left(\beta_{k}\right) E_{\alpha_{k}, \beta_{k}}\left(\frac{\varepsilon_{k}}{G_{0_{k}}}\left(\frac{2-2 \xi_{k}}{2-\xi}\right) \sigma_{x x_{k}}\right)} .
\end{gathered}
$$

We have defined $\boldsymbol{\alpha}=\left(\alpha_{1}, \alpha_{2}, \ldots, \alpha_{N}\right), \boldsymbol{\beta}=\left(\beta_{1}, \beta_{2}, \ldots, \beta_{N}\right), \boldsymbol{\lambda}=\left(\lambda_{1}, \lambda_{2}, \ldots, \lambda_{N}\right)$, $\boldsymbol{\xi}=\left(\xi_{1}, \xi_{2}, \ldots, \xi_{N}\right), \boldsymbol{G}_{\mathbf{0}}=\left(G_{0_{1}}, G_{0_{2}}, \ldots, G_{0_{N}}\right), \boldsymbol{\varepsilon}=\left(\varepsilon_{1}, \varepsilon_{2}, \ldots, \varepsilon_{N}\right)$. Note that $\boldsymbol{\sigma}_{\boldsymbol{x} \boldsymbol{x}}=\left(\sigma_{x x_{1}}, \sigma_{x x_{2}}, \sigma_{x x_{3}}, \sigma_{x x_{4}}, \sigma_{x x_{5}}, \sigma_{x x_{6}}\right)$, where each $\sigma_{x x_{k}}$ is obtained from Eq. (10) for the given $\dot{\gamma}$. 


\section{Optimisation Problems}

The parameters $\boldsymbol{\alpha}, \boldsymbol{\beta}, \boldsymbol{\lambda}, \boldsymbol{\xi}, \boldsymbol{G}_{\mathbf{0}}$, and $\boldsymbol{\varepsilon}$ are unknown, but can be estimated by minimising two objective functions that measure the error given by the distance between experimental values and model predicted values, over the $\omega$ and the $\dot{\gamma}$ ranges.

Thus, to estimate the model parameters that come from two distinct experiments, we propose a two-step optimisation procedure.

First, we estimate $\boldsymbol{\lambda}$ and $\boldsymbol{G}_{\mathbf{0}}$ parameters from the storage and loss moduli data (problem P1). Then, the optimal values $\boldsymbol{\lambda}^{*}$ and $\boldsymbol{G}_{\mathbf{0}}{ }^{*}$ are used in a second step to estimate $\boldsymbol{\alpha}, \boldsymbol{\beta}, \boldsymbol{\varepsilon}$ and $\boldsymbol{\xi}$ from the shear viscosity data (problem P2).

- (P1): the $\boldsymbol{\lambda}$ and $\boldsymbol{G}_{\mathbf{0}}$ parameters estimation problem is formulated as:

$$
\begin{aligned}
\underset{\boldsymbol{\lambda}, \boldsymbol{G}_{\mathbf{0} \in R^{N}}}{\min } E_{G^{\prime}, G^{\prime \prime}}\left(\boldsymbol{\lambda}, \boldsymbol{G}_{\mathbf{0}}\right) & =\sum_{l}^{N_{G^{\prime} G^{\prime \prime}}}\left(\left[\frac{\hat{G}^{\prime}(\omega)_{l}}{G_{l}^{\prime}}-1\right]^{2}+\left[\frac{\hat{G}^{\prime \prime}(\omega)_{l}}{G_{l}^{\prime \prime}}-1\right]^{2}\right) \\
\text { subject to }: & \lambda_{k}>0, G_{0_{k}}>0, k=1, \ldots, N \\
& \lambda_{k}<\lambda_{k+1}, k=1, \ldots, N-1 \\
& G_{0_{k}} \geq G_{0_{k+1}}, k=1, \ldots, N-1
\end{aligned}
$$

with $N_{G^{\prime} G^{\prime \prime}}$ the number of experimental values obtained for storage $\left(G_{l}^{\prime}\right)$ and loss $\left(G_{l}^{\prime \prime}\right)$ moduli.

- (P2): the $\boldsymbol{\alpha}, \boldsymbol{\beta}, \boldsymbol{\xi}$ and $\boldsymbol{\varepsilon}$ parameters estimation problem is formulated as:

$$
\begin{aligned}
& \min _{\boldsymbol{\alpha}, \boldsymbol{\beta}, \boldsymbol{\varepsilon}, \boldsymbol{\xi} \in R^{N}} E_{\eta}(\boldsymbol{\alpha}, \boldsymbol{\beta}, \boldsymbol{\varepsilon}, \boldsymbol{\xi})=\sum_{l}^{N_{\eta}}\left[\frac{\hat{\eta}\left(\dot{\gamma}, \sigma_{x x}\right)_{l}}{\eta_{l}}-1\right]^{2} \\
& \text { subject to }: \\
& \begin{aligned}
& h\left(\dot{\gamma}_{l} ; \sigma_{x x_{k l}}, \lambda_{k}^{*}, G_{0_{k}}^{*}, \alpha_{k}, \beta_{k}, \varepsilon_{k}, \xi_{k}\right)=0, \quad k=1, \ldots, N, l=1, \ldots, N_{\eta} \\
& \alpha_{k} \beta_{k}, \varepsilon_{k},>0, k=1, \ldots, N \\
& 0 \leq \xi_{k}<1, k=1, \ldots, N
\end{aligned}
\end{aligned}
$$

where we set $\boldsymbol{\lambda}=\boldsymbol{\lambda}^{*}$ and $\mathbf{G}_{\mathbf{0}}=\mathbf{G}_{\mathbf{0}}^{*} \cdot N_{\eta}$ is the number of experimental values obtained for shear viscosity $\left(\eta_{l}\right)$.

\section{Methodologies and Numerical Experiments}

\subsection{Methodologies}

To solve the optimisation problem P1 we have used the Ipopt solver that is available online on the NEOS servers (http://neos.mcs.anl.gov/neos/solvers). The Ipopt (Interior Point optimiser) is a solver for large-scale nonlinear programming (NLP) which implements an interior point line search filter method. The mathematical details of the method can be found in [10-14]. 
Since the problem P2 involves $N \times N_{\eta}$ nonlinear equations (Eq. (10)), this problem is hard to solve. Thus, in this work we propose a Sequential Approach for solving P2. Using a Sequential Approach the optimisation is carried out in the space of the input parameters only. In the Sequential Approach the nonlinear equations and the optimisation problem are solved sequentially, one after the other, giving rise to the bound constrained optimisation problem P2\#.

- (P2\#): the new $\boldsymbol{\alpha}, \boldsymbol{\beta}, \boldsymbol{\xi}$ and $\boldsymbol{\varepsilon}$ parameters estimation problem is formulated as described next (Fig. 3). We now consider the minimisation problem:

$$
\begin{aligned}
\min _{\boldsymbol{\alpha}, \boldsymbol{\beta}, \boldsymbol{\varepsilon}, \boldsymbol{\xi} \in R^{N}} E_{\eta}(\boldsymbol{\alpha}, \boldsymbol{\beta}, \boldsymbol{\varepsilon}, \boldsymbol{\xi}) & =\sum_{l}^{N_{\eta}}\left[\frac{\hat{\eta}\left(\dot{\gamma}, \sigma_{x x}\right)_{l}}{\eta_{l}}-1\right]^{2} \\
\text { subject to } & : \alpha_{k}, \beta_{k}, \varepsilon_{k},>0, k=1, \ldots, N \\
& 0 \leq \xi_{k}<1, k=1, \ldots, N
\end{aligned}
$$

and the nonlinear equations are solved numerically using an iterative procedure, so that the objective function can be evaluated. Thus, the nonlinear equations are satisfied at each iteration of the optimisation process. We note this strategy may lead to a computational demanding process since the nonlinear equations are solved for each iteration of the NLP algorithm. Since the experimental data for the first and second normal stress differences were

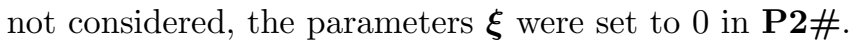

We implement the proposed sequential approach in Matlab ${ }^{\circledR}$, and we use the fmincon function [15-19], to solve the nonlinear problem P2\#. The nonlinear equations were solved using the fsolve function [15]. The sequential optimisation can be described schematically as shown in Fig. 3:

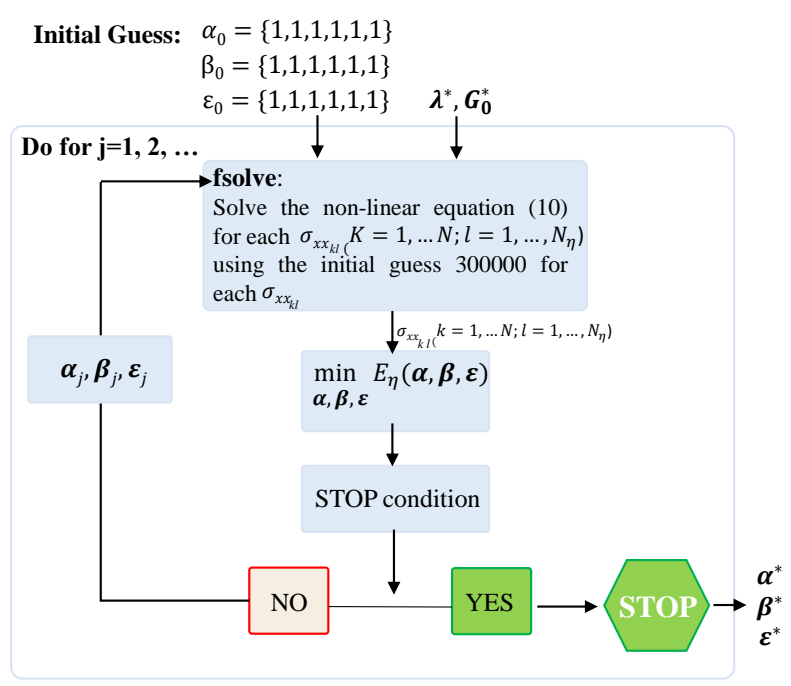

Fig. 3. Algorithm used to solve problem P2\#. 
The experimental data was obtained for a High Density Polyethylene (HDPE) at $190^{\circ} \mathrm{C}$ [9], and is shown in Fig. 4 (symbols) with $N_{G^{\prime} G^{\prime \prime}}=34$ and $N_{\eta}=39$.

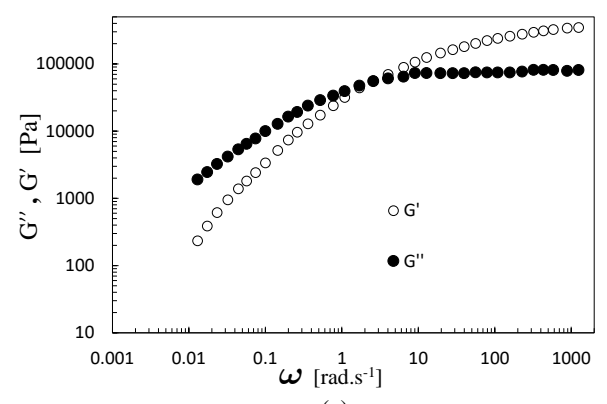

(a)

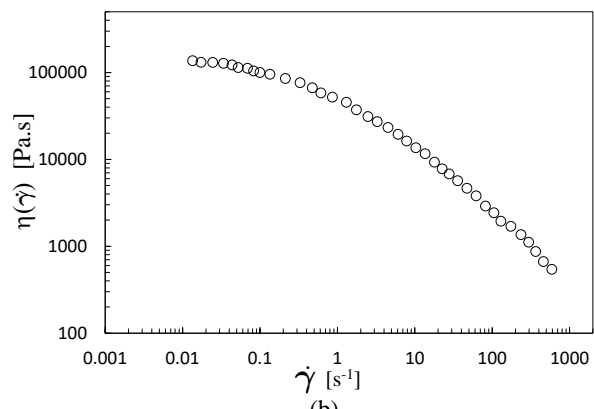

(b)

Fig. 4. Experimental data for a High Density Polyethylene (HDPE) at $190^{\circ} \mathrm{C}$ [9]. (a) Storage and loss moduli; (b) Shear viscosity.

\subsection{Fit to experimental data (storage and loss moduli)}

To obtain the optimal parameters $\boldsymbol{\lambda}^{*}$ and $\boldsymbol{G}_{0}^{*}$ the optimisation problem P1 was coded in AMPL - Modelling Language for Mathematical Programming. By default, AMPL applies a presolve phase to the optimisation problem in order to reduce the size of the problem sent to the solver. Since the decisions made by the presolve phase rely on numerical computations, sometimes small differences between numbers can have a large effect on the results. Thus, to avoid this issue the presolve was disabled.

After some numerical experiments we realised that a value of $N=6$ with initial guess:

$$
\begin{aligned}
& \boldsymbol{\lambda}=(0.005,0.05,0.5,0.9,5,50), \\
& \boldsymbol{G}_{\mathbf{0}}=(100000,85000,70000,40000,10000,1000),
\end{aligned}
$$

could provide good results. We used the IPOPT solver with the default settings and we fed the solver with these initial guess values.

The optimal parameter values $\boldsymbol{\lambda}^{*}$ and $\mathbf{G}_{0}^{*}$ obtained after the optimisation are:

$$
\begin{aligned}
& \lambda^{*}=(0.00113292,0.0112355,0.0882049,0.576664,3.60712,28.496), \\
& \boldsymbol{G}_{0}^{*}=(144113,101039,101039,55374.8,15286.3,1625.3),
\end{aligned}
$$

with $E_{G^{\prime}, G^{\prime \prime}}^{*}=0.104239$. This optimal solution was obtained after 25 objective function evaluations, 25 objective gradient evaluations, 25 inequality constraint evaluations, 25 inequality constraint Jacobian evaluations and 24 Lagrangian Hessian evaluations. The total CPU seconds in IPOPT (without function evaluations) was 0.041 and the total CPU seconds in NLP function evaluations was 0.065 (these computations were performed in the free NEOS computation cloud).

From Fig. 5 we can see that a very good fit was obtained, as expected from the low error value obtained, $E_{G^{\prime}, G^{\prime \prime}}^{*}=0.104239$. 


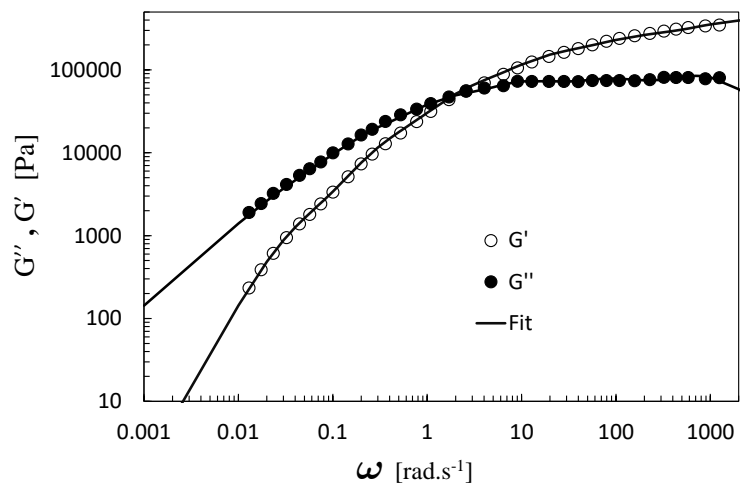

Fig. 5. Experimental data for the storage and loss moduli (symbols) and fit obtained with a 6 -mode gPTT model. The fit was performed by solving problem P1.

\subsection{Fit to experimental data (shear viscosity)}

To obtain the optimal parameters $\boldsymbol{\alpha}^{*}, \boldsymbol{\beta}^{*}, \boldsymbol{\varepsilon}^{*}$ the optimisation problem $\mathbf{P} 2$ \# was solved using the sequential approach described in Fig. 3 and coded in Matlab ${ }^{\circledR}$.

Since the Mittag-Leffler function is not available in the installation package of Matlab ${ }^{\circledR}$, we have used the routine developed by Igor Podlubny and Martin Kacenak (2001-2012).

The fmincon function was used with the interior-point method, considering for stop condition the tolerance $1 \times 10^{-9}$. The remaining parameters were set to default. The fsolve function was used with the trust-region dogleg algorithm [15], where each iteration involves the approximate solution of a large linear system using the method of Preconditioned Conjugate Gradients.

The program stopped after 6 iterations ( $j=6$ - see Fig. 3$)$, resulting in the following optimal parameters:

$$
\begin{aligned}
& \boldsymbol{\alpha}^{*}=(2.8249,1.4277,0.8429,0.6087,1.1905,2.5488), \\
& \boldsymbol{\beta}^{*}=(2.8384,2.6732,3.8326,3.9366,2.9260,3.0454), \\
& \boldsymbol{\varepsilon}^{*}=(3.7370,4.0484,2.8484,2.1562,3.0511,3.3780) .
\end{aligned}
$$

with an error $E_{\eta}=0.3814$. This error is again quite small and therefore a very good fit was obtained (Fig. 6). 


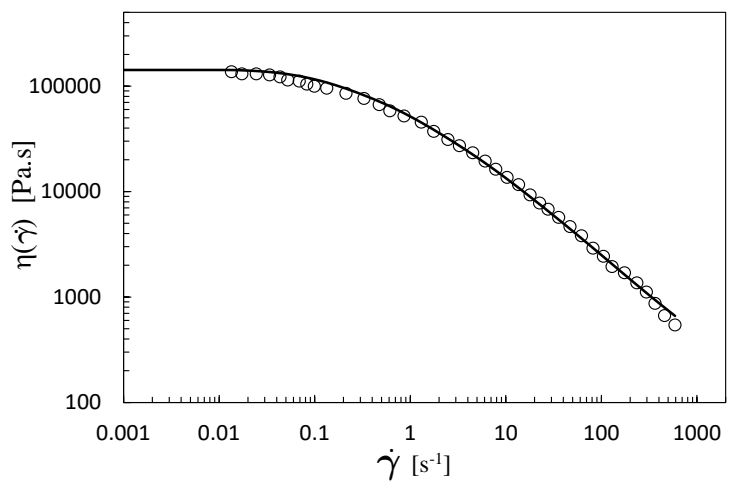

Fig. 6. Experimental data for the shear viscosity (symbols) and fit obtained with a 6-mode gPTT model. The fit was performed by solving problem P2\#.

\section{Conclusions}

In this paper, we propose a two-set optimisation procedure for estimating the parameters of the differential gPTT constitutive equation, that better fit the experimental data obtained for an $\mathrm{HDPE}$ at $190^{\circ} \mathrm{C}$ [9].

First, we estimate $\boldsymbol{\lambda}$ and $\boldsymbol{G}_{\mathbf{0}}$ parameters from the storage and loss moduli data. Then, the optimal values $\boldsymbol{\lambda}^{*}$ and $\boldsymbol{G}_{\mathbf{0}}{ }^{*}$ are used in a second step to estimate $\boldsymbol{\alpha}, \boldsymbol{\beta}$ and $\varepsilon$ from the shear viscosity data.

To obtain the optimal parameter values we formulated two optimisation problems. One to fit the storage and loss moduli data, and the other to fit the shear viscosity data.

These problems were solved using the IPOPT solver and a Sequential Approach, respectively. A very good fit was obtained for both sets of experimental data.

The research group is now considering to optimise all parameters simultaneously, and using a larger set of experimental data.

\section{References}

1. Holzapfel, G. (2001). Nonlinear Solid Mechanics. A Continuum Approach for Engineering.

2. Phan-Thien, N., Tanner, R.I.: New constitutive equation derived from network theory. Journal of Non-Newtonian Fluid Mechanics 2(4), 353-365 (1977)

3. Phan-Thien, N.: A nonlinear network viscoelastic model, Journal of Rheology 22(3), 259-283 (1978).

4. Tanner, R.I., Huilgol, R.R.: On a classification scheme for flow fields. Rheologica Acta 14(11), 959-962 (1975)

5. Ferrás, L.L., Morgado, M.L., Rebelo, M., Mckinley, G.H., Afonso, A.: A generalized Phan-Thien - Tanner model. Journal of Non-Newtonian Fluid Mechanics 269, 88$99(2019)$ 
6. Podlubny, I.: Fractional differential equations: an introduction to fractional derivatives, fractional differential equations, to methods of their solution and some of their applications. Vol. 198. Elsevier (1998)

7. Huilgol, R.R., Phan-Thien, N.: Fluid Mechanics of viscoelasticity: general principles, constitutive modelling, analytical and numerical techniques. Elsevier, Amsterdam (1997)

8. Alves, M.A., Pinho, F.T., Oliveira P.J.: Study of steady pipe and channel flows of a single-mode Phan-Thien - Tanner fluid. Journal of Non-Newtonian Fluid Mechanics 101(1-3), 55-76 (2001)

9. Ansari, M., Hatzikiriakos S.G., Mitsoulis E.: Slip effects in HDPE flows. Journal of Non-Newtonian Fluid Mechanics 167, 18-29 (2012)

10. Nocedal, J., Wächter, A., Waltz, R.A: Adaptive barrier strategies for nonlinear interior methods. SIAM Journal on Optimization 19(4), 1674-1693 (2009)

11. Wächter, A., Biegler, L.T.: Line search filter methods for nonlinear programming: Local convergence. SIAM Journal on Optimization 16(1), 32-48, 2005.

12. Wächter, A., Biegler, L.T.: Line search filter methods for nonlinear programming: Motivation and global convergence. SIAM Journal on Optimization, 16(1), 1-31 (2005)

13. Wächter, A., Biegler, L.T.: On the implementation of a primal-dual interior point filter line search algorithm for large-scale nonlinear programming. Mathematical Programming, 106(1), 25-57 (2006)

14. Wächter, A.: $\mathrm{n}$ interior point algorithm for large-scale nonlinear optimization with applications in process engineering. PhD thesis. Carnegie Mellon University, Pittsburgh, PA, USA (2002)

15. Coleman, T.F., Li, Y.: An interior trust region approach for nonlinear minimization subject to bounds. SIAM Journal on Optimization 6(2), 418-445 (1996)

16. Gill, P.E., Murray, W., Wright, M.H.: Practical Optimization. Academic Press, London (1981)

17. Han, S.P.: A globally convergent method for nonlinear programming. Journal of Optimization Theory and Applications 22(3), 297-309 (1977)

18. Powell, M.J.D.: A fast algorithm for nonlinearly constrained optimization calculations. In: Watson G.A. (eds) Numerical Analysis, Lecture Notes in Mathematics, vol. 630, pp. 144-157. Springer, Berlin, Heidelberg (1978).

19. Powell, M.J.D., The convergence of variable metric methods for nonlinearly constrained optimization calculations. Nonlinear Programming 3, pp. 27-63. Academic Press (1978) 